\title{
Von praxisrelevantem Know-how bis zu innovativem Spezialwissen: IMR 2018
}

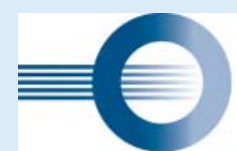

AG Bildgebende Verfahren des Bewegungsapparates

in der Deutschen Röntgengesellschaft

Interview mit Prof. Dr. Marc Regier (München), Vorsitzender der AG Bildgebende Verfahren des Bewegungsapparates und wissenschaftlicher Leiter des Intensivkurses Muskuloskelettale Radiologie (IMR), der am 14. und 15. September 2018 in Hamburg stattfindet.

Herr Professor Regier, im September 2018 findet bereits zum zweiten Mal der Intensivkurs Muskuloskelettale Radiologie (IMR) statt. Wenn Sie noch einmal auf die Premiere 2017 zurückblicken - Worauf können Sie in diesem Jahr aufbauen und was zeichnet einen Intensivkurs im Vergleich zu anderen Fortbildungsveranstaltungen aus?

Der Intensivkurs Muskuloskelettale Radiologie im September 2017 ist die erste Veranstaltung, die von der DRG-Arbeitsgemeinschaft Bildgebende Verfahren des Bewegungsapparates in dieser Form ausgerichtet wurde und mit 150 Teilnehmern ausgebucht war. Unser Ziel war es, den an der muskuloskelettalen Diagnostik interessierten Teilnehmern eine Balance zwischen praxisrelevantem Basiswissen, Protokollempfehlungen für die tägliche Routine und innovativem Spezialwissen zu offerieren. Gleichzeitig wollten wir den Dialog mit den klinischen Fächern intensivieren, weshalb auch dieses Jahr in zahlreichen Sitzungen jeweils ein klinischer Referent darüber berichten wird, welche Antworten er sich von uns Radiologen erhofft und was für unsere Patienten wirklich behandlungsrelevant ist.

Welche Schwerpunktthemen haben Sie für den diesjährigen IMR gesetzt und warum?

Wir werden uns dieses Jahr im Besonderen vier Schwerpunktthemen widmen. Dabei steht neben der Diagnostik der Knochentumoren und dem praxisrelevanten Wissen zu putriden und non-putriden Entzündungen insbesondere die oftmals von vielen etwas gefürchtete Bildgebung von Ellenbogen, Hand- und Fingergelenken sowie OSG und Vorfuß im Vordergrund. Als vierten Schwerpunkt haben wir auch dieses Jahr wieder einen Fokus auf die typischen Verletzungsmuster in der Sportmedizin gelegt. In diesem sich rasch entwickelnden Teilgebiet der muskuloskelettalen Diagnostik werden von uns Radiologen besondere, sportassoziierte Kenntnisse erwartet, welche wir den Teilnehmern dieses Jahr und auch zukünftig auf dem IMR als ein Schwerpunktthema vermitteln möchten.

Können Sie schon ein besonderes Highlight verraten, das die Teilnehmerinnen und Teilnehmer erwartet?

Allzu viel möchte ich noch nicht verraten; auf einen Highlight-Vortrag zum Thema Knochentumoren bin ich persönlich allerdings schon heute gespannt. Ich freue mich deshalb sehr auf Herrn Professor Maximilian Reiser, der sicher jedem Radiologen bekannt und ein herausragender Referent ist. Im großen Helmut-SchmidtAuditorium im Herzen Hamburgs werden uns die mehr als 20 Referenten auf den neuesten Wissensstand bringen, bevor wir am Freitagabend die Geselligkeit nicht vernachlässigen und zusammen in der Hamburger Elbphilharmonie den Tag ausklingen lassen wollen.

Haben Sie auch für $\mathbf{2 0 1 8}$ wieder einen besonderen Überraschungsgast eingeplant?

Selbstverständlich! Im letzten Jahr haben wir ja mit Marcel Jansen einen ehemaligen Fußballnationalspieler vom HamburgerSport-Verein als Gast begrüßen dürfen. Er stand den Teilnehmern vor dem Hintergrund der zunehmenden Bedeutung der medizinischen Versorgung im Profisport während und auch nach der Veranstaltung ausführlich Rede und Antwort. Auch in diesem Jahr dürfen wir uns wieder auf einen prominenten Sportler freuen, der uns eini-

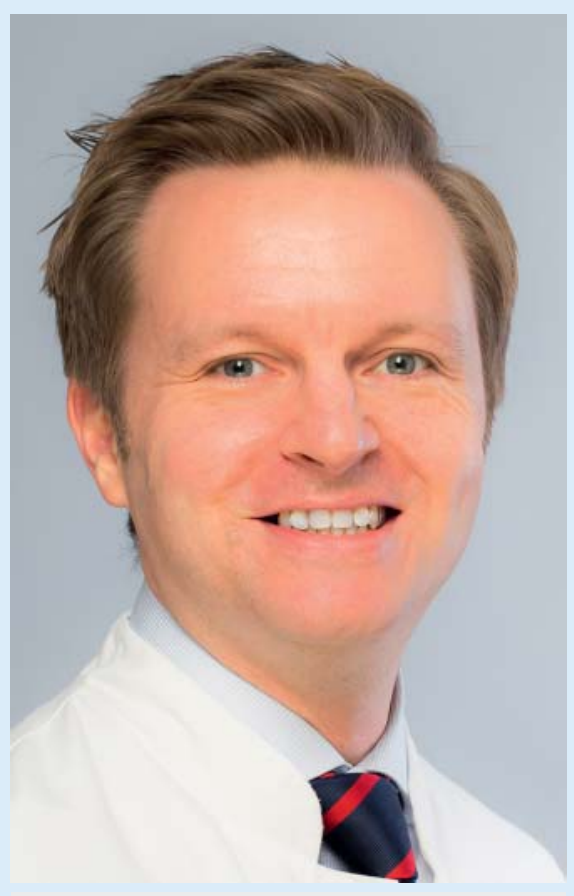

Prof. Dr. Marc Regier

ge interessante Einblicke in die Schlüsselmomente seiner Karriere geben soll. Er wird zudem die Preise an die Gewinner/-innen des TED-Quiz überreichen und für ein Meet-and-Greet bereitstehen. Um wen genau es sich dabei handelt, möchte ich noch nicht verraten.

Welche persönlichen Erwartungen knüpfen Sie an den zweiten IMR?

Ich hoffe auf eine interessante und inspirierende Veranstaltung, die allen Teilnehmern eine Auffrischung von Fachwissen und die Vertiefung aktueller Erkenntnisse der vielschichtigen muskuloskelettalen Radiologie ermöglicht und darüber hinaus als ein erinnernswerter Aufenthalt in Hamburg im Gedächtnis bleibt. 